\title{
APPLICABILITY OF THE SURGICAL SITE INFECTION RISK INDEX TO PATIENTS UNDERGOING CARDIAC, NEUROLOGIC AND ORTHOPEDIC SURGERY
}

\author{
Débora Marie da Silva Bonmann'1, Carem Gorniak Lovatto², \\ Caroline Deutschendorf ${ }^{2}$
}

\begin{abstract}
Introduction: The surgical site infection risk index (SSIRI) includes three risk factors for surgical site infection (SSI): potential for surgical contamination, duration of surgery, and patient's ASA score. A patient having a preoperative American Society of Anesthesiologists (ASA) score of III, IV or IV; a surgery classified as either contaminated or dirty-infected; an operation with duration of surgery more than T hours, where T depends on the surgical procedure being performed are considered in risk of SSI. The SSIRI can be used as a method of postoperative surveillance. The aim of this study is to analyze the applicability of the SSIRI to patients undergoing cardiac, neurologic and orthopedic surgery.
\end{abstract}

Methods: A retrospective cohort study including patients undergoing cardiac, neurological and orthopedic surgeries considered major, from January to December 2014 , evaluated through review of medical charts of patients admitted to a university hospital in southern Brazil. Values of $p<0.05$ were considered significant.

Results: A total of 761 patients were included in the study; of these, $122(16 \%)$ developed SSI. The SSIRI did not demonstrate statistical significance to distinguish the patients most likely to develop SSI between the four levels of the ASA score. Black and brown patients, patients who underwent intraoperative blood transfusion, and patients who underwent surgical reintervention were more susceptible to SSI.

Conclusions: The SSIRI could not be used as a method of postoperative surveillance for patients in the present study. The other risk factors found may serve as a guide to articulate SSI prevention strategies and, thus, minimize chances of development of serious infections.

Keywords: Postoperative complications; infection; risk index

Surgical site infections (SSIs) are defined as healthcare associated infections resulting from complications of surgery that cause significant morbidity and mortality and increase healthcare costs ${ }^{1}$. In Brazil, they are the third leading cause of nosocomial infections ${ }^{2}$. SSIs may be classified into superficial incisional, deep incisional, or organ/cavity, according to the depth to which they affect the tissues, are considered to result from a surgical procedure, and usually manifest within the first 30 days after surgery or after 1 year, in case of placement of orthoses or prostheses. The criteria commonly used to identify SSI are: drainage of purulent fluid; culture-positive aseptic fluids or tissues; presence of surgical wound abscess; surgical reintervention; clinical, histopathological or imaging examination; diagnosis of infection by the primary physician ${ }^{3}$. The deeper the SSI, the worse the consequences for the patient, including the possibility of death. Therefore, it is important to evaluate methods of postoperative surveillance for the occurrence of SSI so as to develop strategies of prevention and early identification.

Clin Biomed Res. 2017;37(2):81-86

1 Integrated Multidisciplinary Residency in Health, Hospital de Clínicas de Porto Alegre (HCPA). Porto Alegre, RS, Brazil.

2 Hospital Infection Control Committee, Hospital de Clínicas de Porto Alegre (HCPA). Porto Alegre, RS, Brazil.

Corresponding author:

Débora Marie da Silva Bonmann deborabonmann89@gmail.com Residência Integrada Multiprofissional em Saúde, Hospital de Clínicas de Porto Alegre (HCPA)

Rua Ramiro Barcelos, 2350

90035-903, Porto Alegre, RS, Brazil. 
The SSI risk index (SSIRI) is a postoperative surveillance method designed by the National Nosocomial Infections Surveillance that includes three risk factors for SSI, namely: potential of contamination (contaminated or dirty-infected surgeries score one point); surgical duration (surgeries longer than the 75th percentile of duration of surgery score one point); and risk classification according to the American Society of Anesthesiology (ASA) (patients classified as ASA III, IV or V score one point). In a validation study of this index, SSI rates for patients with SSIRI scores of $0,1,2$ and 3 were $1.5 \%, 2.9 \%, 6.8 \%$, and $13.0 \%$ of surgeries, respectively ${ }^{4}$. That is, each additional risk factor increases the likelihood of SSI.

The SSIRI is widely used in several countries, but has not been validated for the Brazilian population yet. ${ }^{5}$ In our hospital, the SSIRI is not systematically used. Thus, the present study aimed to evaluate a new method of searching for SSI, since this complication is of great epidemiological importance, and the consequences for the patient may be exacerbated by the delay in receiving diagnosis and treatment. If there is a good correlation between the SSIRI and SSIs in patients who underwent cardíac, neurological, and orthopedic surgeries, the follow-up of patients with the greatest number of risk factors for developing SSI may be improved, which prevent losses and justifies the present study to evaluate the applicability of the SSIRI.

The general aim of this study was to analyze the applicability of the SSIRI to patients of a university hospital in southern Brazil by assessing the direct correlation between the SSIRI and SSI rates in cardiac, neurological, and orthopedic major surgeries conducted in 2014. The specific aim was to analyze other factors that may be associated with the occurrence of SSI that are not included in the calculation of the SSIRI.

\section{METHODS}

A quantitative, observational, retrospective, cohort study approved by the Research Ethics Committee (CEP) under the register number CAAE 52157615.8.0000.5327 was conducted in a university hospital in southern Brazil.

SSIs were identified by active search of patients admitted to the study hospital to monitor their evolution during hospitalization and by active post-discharge search of their postoperative outpatient visits. There is a possibility of loss due to surgeon's failure to notify SSIs and patient's failure to attend postoperative visits.

We reviewed the online medical records of patients meeting inclusion criteria, i.e., who underwent cardiac, neurological, and orthopedic surgeries from January to December 2014. Primary variables (duration of surgery, ASA class, and potential of contamination) were collected for calculating the SSIRI. Secondary variables were gender, ethnicity, age, surgical service, use of orthoses and prostheses, infusion of blood components, surgical reintervention (defined as a new procedure related to the main surgery due to an unfavorable outcome without restriction of time after surgery), surgical status (urgent vs. elective), length of hospital stay before surgery (in the present study, admission greater than 24 hours was considered a risk factor for SSI), and microorganisms isolated from surgical wound cultures. Patients whose medical records did not provide all information required to calculate the SSIRI, patients who died during the intraoperative period or within the first 30 days after surgery (time frame for the development of SSIs), patients classified as ASA VI (brain death organ donor), and patients who remained admitted for less than 30 days and did not return to postoperative visits, making their follow-up impossible, were excluded from the study. We searched data from 30 days after surgeries who did not involve placement of prostheses and orthoses or from 1 year after surgeries for placement of prostheses and orthoses. The diagnosis of SSI was based on the criteria established by the Center for Disease Control and Prevention ${ }^{3}$.

Statistical analysis was made using the Epi Info version 2000 Beta and the Statistical Package for the Social Sciences (SPSS) software version 18.0. Simple frequency distribution, measures of central tendency (mean or median), and measures of variability were obtained to characterize and describe patients. Overall incidence rates were calculated for each SSIRI score, and chi-squared test and ANOVA were used to assess the statistical correlation of the SSIRI with SSI rates and other possible risk factors for SSI. A value of $p<0.05$ was considered statistically significant.

\section{RESULTS}

Out of a population of 1,223 patients, 761 who underwent cardiac, neurological, and orthopedic surgeries were included in the study. The main reasons for exclusion were length of hospital stay below 30 days and failure to attend to outpatient visits, accounting for 394 (85.3\%) cases. Since this is a retrospective study and the study hospital does not routinely conduct post-discharge searches by telephone or questionnaire to investigate postoperative outcomes of all surgical patients, some of them may have developed SSI and may have sought other healthcare facilities, making it impossible for the study institution to know whether the patient developed SSI or not. The other reasons for exclusion from the study 
were death within the first 30 days after surgery, with $63(13.6 \%)$ cases; missing information to calculate the SSIRI, with $3(0.6 \%)$ cases; and intraoperative death, with $2(0.5 \%)$ cases.

Of the 761 patients who underwent surgery, $122(16 \%)$ developed SSI. The most frequent depth of infection was deep incisional, with 49 (40\%) cases, followed by organ/cavity, with $45(37 \%)$ cases, and deep incisional, with $28(23 \%)$ cases. The SSI rates for patients with SSIRI scores of $0,1,2$ e 3 were $247(32.5 \%), 426(56.0 \%), 87(11.4 \%)$, and $1(0.1 \%)$, respectively. SSI infection occurred in $37(15.0 \%)$ patients with SSIRI score of 0, $66(15.5 \%)$ with SSIRI score of $1,19(21.8 \%)$ with SSIRI score of 2, and none of the patients with SSIRI score of 3 .

With regard to the potential of surgical contamination, $726(95.4 \%)$ surgeries were classified as clean, $34(4.5 \%)$ were classified as infected, and $1(0.1 \%)$ was classified as potentially contaminated. As for ASA class, $402(52.8 \%)$ patients were classified as ASA I and II, whereas 359 (47.2\%) were classified as ASA III, IV and V. When it comes to duration of surgery, $213(28 \%)$ surgeries were longer than the 75th percentile for duration of surgery.

Higher SSIRI scores did not lead to a statistically significant increase in the occurrence of SSI. In the present study, the incidence of SSI was higher than that of a previous validation study. Figure 1 shows the correlation between SSI rates and SSIRI scores in the current study and in the validation study.

There was no difference in infection rates stratified by gender and age. The use of prostheses or orthoses, length of hospital stay before surgery, and surgical status exhibited no statistically significant difference in the development of SSI. Similarly, the three surgical services analyzed (cardiac, neurological, and orthopedic surgeries) were not

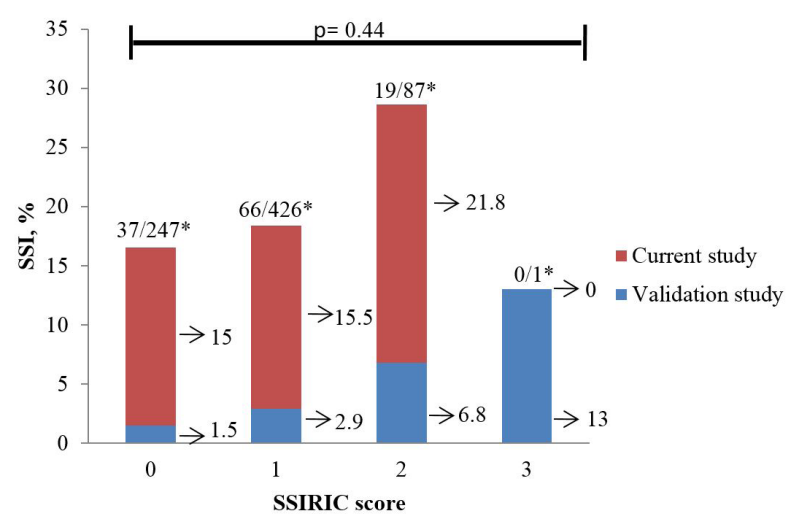

Figure 1: Comparison between surgical site (SSI) rates and SSI risk index (SSIRI) scores. *Number of patients with SSI/Number of patients with the corresponding SSIRI score. risk factors for SSI. In addition, black and brown patients were more likely to develop SSI when compared to white patients (odds ratio $[\mathrm{OR}]=1.9$, $95 \%$ confidence interval $[95 \% \mathrm{Cl}] 1.2-3.1 ; p=0.003$ ). The intraoperative infusion of blood components was found to promote a nearly two-fold increase in the risk of $\mathrm{SSI}(\mathrm{OR}=2.14 ; 95 \% \mathrm{Cl} 1.14-4.02 ; \mathrm{p}=0.01)$, whereas surgical reintervention promoted a nearly seven-fold increase in this risk $(\mathrm{OR}=7.67 ; 95 \% \mathrm{Cl}$ 3.77-15.58; $p=0.01$ ), as shown in Table 1.

With regard to surgical wound cultures, $45(37.0 \%)$ were positive. Gram-positive bacteria accounted for $60.0 \%$ of SSI cases, followed by Gram-negative bacteria, with $37.8 \%$ of the cases, and funguses, with $2.2 \%$ of the cases. The microorganism most commonly isolated from patients with SSI was Staphylococcus aureus, with $16(35.6 \%)$ cases. Table 2 shows a detailed description of the microorganisms isolated from patients with SSI.

\section{DISCUSSION}

The risk factors assessed using the SSIRI were not able to distinguish which patients are more likely to develop SSI, because there was no statistically significant difference in the incidence of SSI between groups of patients with different SSIRI scores. Only one patient had a SSIRI of 3 , but did not develop SSI. This probably results from the low potential of contamination of surgeries, because $95.5 \%$ of them were classified as clean or potentially contaminated, which means that almost all patients did not score in one of the three risk factors for SSI. The other risk factors included in the SSIRI also had a low frequency, since $52.8 \%$ of patients did not present with comorbidities identified as a risk factor for SSI according to the ASA classification, and $72 \%$ of surgeries occurred within an appropriate time period.

Two Brazilian studies 5,6 also found that the SSIRI was not correlated with SSI rates in their study population, and one study ${ }^{7}$ found that there was a statistically significant correlation between SSIRI scores and SSI rates. The first of these studies investigated orthopedic surgical patients, showing that there was a linear increase in the SSI rates as the SSIRI score increase, but, similarly to the present study, this increase was small and had no statistical significance. Additionally, this same study found patients with a SSIRI score of 0, 1 and 2 only, with score 2 accounting for less than $8 \%$ of study patients. No patient was classified into SSIRI score of $3^{5}$. In the second study, which analyzed patients who underwent procedures of 97 surgical services, $97.8 \%$ of procedures scored SSIRI 0 and SSIRI 1, and the incidence of SSI was higher than that expected according to the previous validation study. There 
Table 1: Characterization of patients, surgeries, and development of SSI.

\begin{tabular}{|c|c|c|c|}
\hline Variable & Frequency (n, \%) & SSI (n, \%) & \\
\hline \multicolumn{4}{|l|}{ Gender } \\
\hline Female & $361 / 761(47 \%)$ & $63 / 361(17.4 \%)$ & \multirow{2}{*}{$p=0.15$} \\
\hline Male & $400 / 761(53 \%)$ & $59 / 400(14.7 \%)$ & \\
\hline \multicolumn{4}{|l|}{ Race/ethnicity } \\
\hline Black & $52 / 761(6.8 \%)$ & $15 / 52(28.8 \%)$ & \multirow{3}{*}{$p=0.003$} \\
\hline Brown & $24 / 761(3.2 \%)$ & $6 / 24(25 \%)$ & \\
\hline White & $685 / 761(90 \%)$ & $101 / 685(14.7 \%)$ & \\
\hline \multicolumn{4}{|l|}{ Age, years } \\
\hline$<20$ & $74 / 761(9.7 \%)$ & $14 / 74(19 \%)$ & \multirow{5}{*}{$p>0.05^{*}$} \\
\hline $20-39$ & $112 / 761(14.7 \%)$ & $13 / 112(10.6 \%)$ & \\
\hline $40-59$ & $221 / 761(29 \%)$ & $30 / 221(13.5 \%)$ & \\
\hline $60-79$ & $316 / 761(41.5 \%)$ & $58 / 316(18.3 \%)$ & \\
\hline$>$ 80: & $38 / 761(5 \%)$ & $7 / 38(18.4 \%)$ & \\
\hline \multicolumn{4}{|l|}{ Surgical service } \\
\hline Cardiology & $230 / 761(30.2 \%)$ & $41 / 230(17.8 \%)$ & \multirow{3}{*}{$p>0.05^{\star}$} \\
\hline Neurology & 233/761 (30.6\%) & $39 / 233(16.7 \%)$ & \\
\hline Orthopedics & 298/761 (39.1\%) & $42 / 298(14 \%)$ & \\
\hline \multicolumn{4}{|c|}{ Use of prostheses or orthoses } \\
\hline No & $405 / 761(53.2 \%)$ & $70 / 405(17.3 \%)$ & \multirow{2}{*}{$p=0.15$} \\
\hline Yes & $356 / 761(46.8 \%)$ & $52 / 356(14.6 \%)$ & \\
\hline \multicolumn{4}{|c|}{ Infusion of blood components } \\
\hline Yes & $57 / 761(7.5 \%)$ & $15 / 57(26.3 \%)$ & \multirow{2}{*}{$p=0.01$} \\
\hline No & $704 / 761(92.5 \%)$ & $107 / 704(15.2 \%)$ & \\
\hline \multicolumn{4}{|l|}{ Surgical reintervention } \\
\hline Yes & $34 / 761(4.5 \%)$ & 19/34 (56\%) & \multirow{2}{*}{$p=0.01$} \\
\hline No & $727 / 761(95.5 \%)$ & $103 / 727(14.2 \%)$ & \\
\hline \multicolumn{4}{|l|}{ Surgical status } \\
\hline Urgent & $106 / 761(14 \%)$ & $20 / 106(19 \%)$ & \multirow{2}{*}{$p=0.3$} \\
\hline Elective & $655 / 761(86 \%)$ & $102 / 655(15.6 \%)$ & \\
\hline \multicolumn{4}{|c|}{ Length of hospital stay before surgery } \\
\hline$<24 \mathrm{~h}$ & $405 / 761(53.2 \%)$ & $68 / 405(16.8 \%)$ & \multirow{2}{*}{$p=0.5$} \\
\hline$>24 \mathrm{~h}$ & $356 / 761(46.8 \%)$ & $54 / 356(15.2 \%)$ & \\
\hline
\end{tabular}

*When compared to the group of higher incidence of SSI with the others.

Table 2: Microorganisms isolated from fluid cultures of surgical wounds.

\begin{tabular}{lcc}
\hline \multicolumn{1}{c}{ Microorganism } & $\mathbf{n}$ & $\%$ \\
\hline Gram-positive & & \\
Staphylococcus aureus & 16 & 35.6 \\
Staphylococcus sp coagulase negative & 9 & 20 \\
Enterococcus sp & 1 & 2.2 \\
Streptococcus sp & 1 & 2.2 \\
Gram-negative & & \\
Klebsiella sp & 4 & 9 \\
Enterobacter sp & 4 & 9 \\
Proteus sp & 2 & 4.4 \\
Pseudomonas sp & 2 & 4.4 \\
Serratia sp & 2 & 4.4 \\
Citrobacter sp & 2 & 4.4 \\
Acinetobacter $s p$ & 1 & 2.2 \\
Funguses & & \\
Aspergilus $s p$ & 1 & 2.2 \\
\hline
\end{tabular}

was statistical significance only in gastrointestinal surgeries, cases in which the study correlated the $\mathrm{SRI}$ and SSI according to mefical service ${ }^{6}$. The last study pointed out the correlation between the SRI and SSI rates in clean and potentially contaminated surgeries; however, rates were also higher than those of the validation study ${ }^{7}$.

In the present study, other risk factors for SSI were observed, namely intraoperative transfusion of blood components, surgical reinterventions, and African or mixed ethnicity. Blood transfusion causes patient's immunosuppression, which may have increased the likelihood of developing $\left.S S\right|^{8-10}$. Reintervention may be a risk factor due to additional handling of the surgical site, favoring the colonization and carrying of microorganisms to the surgical site ${ }^{10}$. Ethnicity may pose risks for infection due to the intrinsic risks of this population ${ }^{6}$. 
With regard to the other data collected in the present study that could also be risk factors for SSI, there was no statistically significant difference in the development of SSI in terms of sex, age, surgical service, hospital length of stay before surgery, surgical status, and use of prostheses and orthoses. The correlation between SSI and sex has shown conflicting results. One study found that $\mathrm{SSI}$ is more common in male patients $(p<0.001)^{9}$, whereas another found that it was more common in female patients $(p=0.000)^{11}$. Gastrointestinal surgeries exhibited differences in the development of SSI. The results may be attributed to the risk of microbial colonization in these surgeries ${ }^{8}$. The study by Kok et al. ${ }^{12}$ shows that $19.6 \%$ of patients who underwent an emergency surgery and $6.8 \%$ of those who underwent elective surgery developed SSI. The increased risk of infection in emergency surgeries was attributed to inadequate preoperative optimization of assessment of comorbidities and contamination at the surgical site. The use of prostheses and orthoses has been identified as a risk factor due to the formation of a bacterial biofilm in these devices, which acts as a barrier against the host immune response and antibiotic penetration ${ }^{10}$ and increases microorganism colonization ${ }^{8}$.

Consistent with the literature on depth of infection ${ }^{8}$, incisional infection was the most frequent in the present study ( $40 \%$ of SSI cases). However, more severe organ/cavity infections were the second more frequent in our study ( $37 \%$ of cases of SSI), but the third leading cause in the study by Isik et al., accounting for $18 \%$ of the cases ${ }^{8}$.

With regard to microorganisms isolated from fluid cultures of patients with SSI, other Brazilian study also found that the predominant isolates were Gram-positive microorganisms (53\% of the observed infections). Gram-negative bacilli and funguses accounted for $45 \%$ and $2 \%$ of the isolates, respectively ${ }^{13}$. Conversely, a European study found that Gram-negative isolates were the predominant findings, with $82.7 \%$ of patients with SSI, followed by Gram-positive isolates, with $15 \%$ of patients, and funguses, with $2.3 \%$ of patients ${ }^{8}$. Since Gram-positive microorganisms are commonly found in the skin, it is acceptable that they occur more frequently in surgical wound cultures. However, it bears noting the importance of correct skin antisepsis with chlorhexidine baths before the procedure, antisepsis of surgical wound with alcohol solutions, chlorhexidine, or povidone-iodine (PVP-I), previous decolonization of patients carrying Staphylococcus aureus, and correct cleaning of hands with alcohol or chlorhexidine before incision ${ }^{14}$.

The study may pose risk to patients due to the possible breach of confidentiality resulting from the access to online medical records. Additionally, it revealed the collective benefit of future interventions for patients who underwent preventive surgeries and intervention in postoperative complications. Other studies should be conducted with an increased number of patients and surgical services to investigate the applicability of the SIIRI in other patients with SSI.

The most important limitations of the study lie on its retrospective design, leading to depending on the availability of information in online medical records, and on the types of surgeries listed, which restricted the possibility of including more patients with SIIRI score of 3. Data comparison was complicated by the scarcity of studies analyzing the same topic.

The SSIRI was not applicable to patients and types of surgery assessed in this study, because the sum of risk factors included in this index did not result in significant differences. Conversely, intraoperative transfusion of blood components, surgical reinterventions, and black or mixed ethnicity were shown to be a risk factor for SSI. These factors enable to articulate strategies to prevent SSI, improve methods of active search of signs and symptoms in patients with SSI, and minimize the risk of developing severe infections.

\section{Acknowledgements}

We thank the Hospital Infection Control Committee for providing us with data and for having contributed to the development of this research.

\section{Conflicts of interest}

The authors declare no conflicts of interest.

\section{REFERENCES}

1. Gibbons C, Bruce J, Carpenter $\mathrm{J}$, Wilson AP, Wilson J, Pearson $A$, et al. Identification of risk factors by systematic review and development of risk-adjusted models for surgical site infection. Health Technol Assess. 2011;15(30):1-156, iii-iv. PMid:21884656. http://dx.doi. org/10.3310/hta15300.
2. Brasil. Agência Nacional de Vigilância Sanitária. Critérios Nacionais de Infecções relacionadas à assistência à saúde. Brasília: ANVISA; 2009. Série Segurança do Paciente e Qualidade em Serviços de Saúde.

3. Centers for Disease Control and Prevention (CDC). Protocol corrections, clarification, and additions: procedure-associated events surgical site infection. Atlanta; 2013.

4. Culver DH, Horan TC, Gaynes RP, Martone WJ, Jarvis WR, Emori TG, et al. Surgical wound infection rates by wound class, operative 
procedure, and patient risk index. National Nosocomial Infections Surveillance System. Am J Med. 1991;91(3B):152S-7S. PMid:1656747. http://dx.doi.org/10.1016/00029343(91)90361-Z.

5. Ercole FF, Chianca TCM, Duarte D, Starling CEF, Carneiro M. Infecção de sítio cirúrgico em pacientes submetidos a cirurgias ortopédicas: o índice de risco NNIS e predição de risco. Rev Lat Am Enfermagem. 2011;19:269-76. PMid:21584372. http://dx.doi.org/10.1590/S010411692011000200007.

6. Nogueira JM, Couto RC. Reprodutibilidade do índice de risco para infecção em sítio cirúrgico do National Nosocomial Infections Surveillance System (CDC, USA) em hospitais de Belo Horizonte MG [dissertação]. Belo Horizonte (MG): Universidade Federal de Minas Gerais; 2009.

7. Santos ML, Teixeira RR, Diogo-Filho A. Surgical site infections in adults patients undergoing of clean and contaminated surgeries at a university Brazilian hospital. Arq Gastroenterol. 2010;47(4):383-7. PMid:21225150. http://dx.doi.org/10.1590/S000428032010000400012.

8. Isik O, Kaya E, Dundar HZ, Sarkut P. Surgical site infection: re-assessment of the risk factors. Chirurgia. 2015;110(5):457-61.

9. Moreno Elola-Olaso A, Davenport DL, Hundley JC, Daily MF, Gedaly R. Predictors of surgical site infection after liver resection: a multicentre analysis using National Surgical Quality Improvement Program data. HPB (Oxford). 2012;14:136-41.

10. Parchi PD, Evangelisti G, Andreani L, Girardi F, Darren L, Sama A, et al. Postoperative spine infections. Orthop Rev. 2015;7(3):5900. PMid:26605028. http://dx.doi.org/10.4081/or.2015.5900.

11. Kim JS, Kim SK, Kang DH, Hwang $\mathrm{SH}$, Jung JM, Han JW. Analysis of the risk factors affecting the surgical site infection after cranioplasty following decompressive craniectomy. Korean
J Neurotrauma. 2015;11(2):1005. PMid:27169073. http://dx.doi. org/10.13004/kjnt.2015.11.2.100.

12. Kok TWK, Agrawal N, Sathappan SS, Chen WK. Risk factors for early implant-related surgical site infection. J Orthop Surg (Hong Kong). 2016;24(1):72-6. PMid:27122517. http://dx.doi.org/10.1177/23094990160 2400117.

13. Oliveira PR, Carvalho VC, Felix CS, Paula AP, Santos-Silva J, Lima ALLM. The incidence and microbiological profile of surgical site infections following internal fixation of closed and open fractures. Rev Bras Ortop. 2016;51(4):396-9. PMid:27517016. http://dx.doi.org/10.1016/j. rbo.2015.09.010.

14. World Health Organization (WHO). Global guidelines for the prevention of surgical site infection. Geneva: WHO; 2016 [cited 2017 June 19]. Available from: http://apps.who.int/iris/bitstre am/10665/250680/1/9789241549882eng.pdf?ua= 